\title{
Monte Carlo transport simulation for a Long Counter neutron detector employed as a monitor of cosmic-ray induced neutron at ground level
}

\section{M.T. Pazianotto ${ }^{* \dagger}$}

Departmento de Física, Instituto Tecnológico de Aeronáutica, São José dos Campos SP

E-mail: mpazianotto@gmail.com

\section{O.L. Gonçalez}

Instituto de Estudos Avançados, EFA, Departamento de Ciência e Tecnologia Aeroespacial, São José dos Campos SP

E-mail: odairleieav.cta.br

\section{C.A. Federico}

Instituto de Estudos Avançados, EFA, Departamento de Ciência e Tecnologia Aeroespacial, São

José dos Campos SP

E-mail: claudiofederico@ieav.cta.br

\section{B.V. Carlson}

Departamento de Física, Instituto Tecnológico de Aeronáutica, São José dos Campos SP

E-mail: bretteita.br

The objective of this work is to present the model used to simulate an isotropic neutron source with a wide energy spectrum extending up to high energy employing the MCNPX code (Monte Carlo N-Particle eXtended) and then discuss the LC efficiency employing this neutron source. The calculated LC efficiency is compared with experimental results for cosmic-ray neutron measurements at ground level.

XXXIV edition of the Brazilian Workshop on Nuclear Physics,

5-10 June 2011

Foz de Iguaçú, Paraná state, Brasil

\footnotetext{
*Speaker.

${ }^{\dagger}$ MTP would like to thank CAPES for financial support. BVC acknowledges support from FAPESP and the CNPq.
} 


\section{Introduction}

High energy neutrons are produced by interactions between primary cosmic rays and atmospheric atoms and undergo moderation, resulting in a wide spectrum of energy ranging from thermal energies $(0.025 \mathrm{eV})$ to energies of several hundreds of $\mathrm{MeV}$. Measurements of the cosmic radiation dose on-board aircraft need to be followed with an integral flow monitor at ground level in order to register CR intensity variations during the measurements. The Long Counter (LC) neutron detector ([1],[2],[3]) was designed as a directional neutron flux meter standard because it presents fairly constant response for energy under $10 \mathrm{MeV}$. However we would like to use it as a ground-based neutron monitor for the cosmic-ray induced neutron spectrum (CRINS) that presents an almost isotropic fluency and a wider spectrum of energy. In previous work ([4], [5], [6]), the LC was modeled and tested using a Monte Carlo transport simulation for irradiation with known neutron sources $\left({ }^{241} \mathrm{Am}-\mathrm{Be}\right.$ and $\left.{ }^{252} \mathrm{Cf}\right)$ as a benchmark in order to assure the geometric description of the LC using MCNPX input data.

To model the LC response to the cosmic-ray induced neutron spectrum, one must understand the LC efficiency for an almost isotropic flux. Using the model geometric described below, the efficiency was calculated for a CRINS isotropic flux including high energy neutron interaction models.

The objective of this work is to present the model used to simulate an isotropic neutron source with a wide energy spectrum extending up to high energy employing the MCNPX code [7] (Monte Carlo N-Particle eXtended) and then discuss the LC efficiency employing this neutron source. The calculated LC efficiency is compared with experimental results for cosmic-ray neutron measurements at ground level.

\section{Methodology}

The simulation was performed by modelling the following experimental arrangement: The LC was assumed to be subject to a cosmic-ray induced neutron spectrum over a $2 \pi$ steradians source. [5]

We used two approaches to reproduce the LC irradiated by this source:

a) Successive simulations were performed using a point isotropic source placed along a meridian at various increasing angles, ranging from zenith ( 0 degree) to the horizon, and the results of each simulation were combined as the average of the integration over the $2 \pi$ steradians solid angle.

b) Simulations were perfomred using as MCNPX input a semi-spherical source for which all particle emissions converge at the center point at which the LC is located, as presented in Figure 1.

In the first approach, the simulations were assumed to use a point neutron source at a distance of $1.66 \mathrm{~m}$ from the geometric center of the LC for the following angles: 0, 15, 30, 45, 60, 75 and 90 degrees. The LC efficiency is defined as the ratio between the number of capture reactions of thermal neutrons by ${ }^{3} \mathrm{He}$ counted in the central detector and the incident flux on the LC surface. 


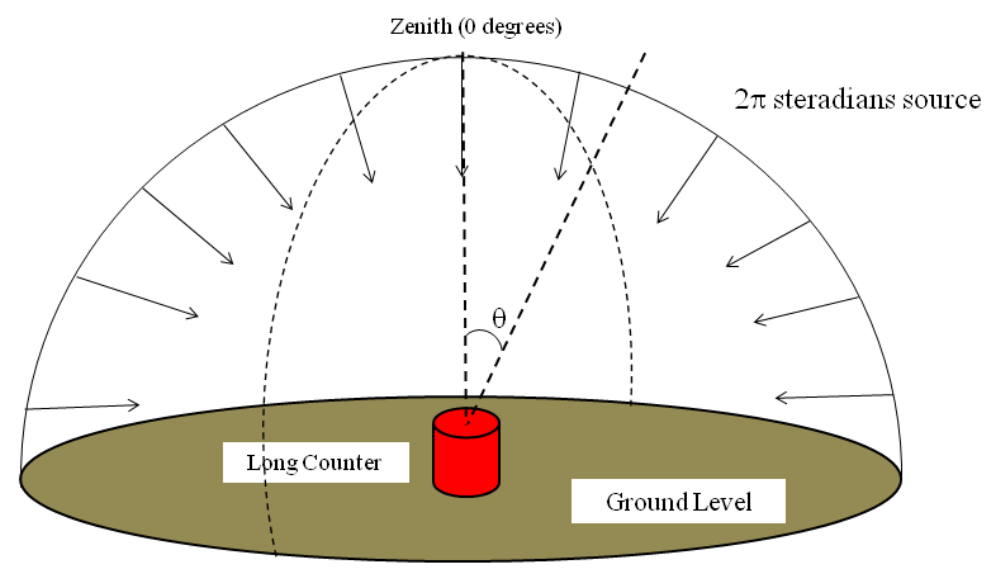

Figure 1: Representative drawing of the experimental setup used in the simulation.

In the second approach, the Long Counter efficiency was computed in the same manner. The neutron sources were modelled using the MCNPX code for the cosmic-ray induced neutron spectrum measured at Itatiaia Mountain (2400 km altitude), in Brazil [8] shown in Figure 2. One can see three peaks in the spectrum, associated with its thermal, evaporation and spallation components.

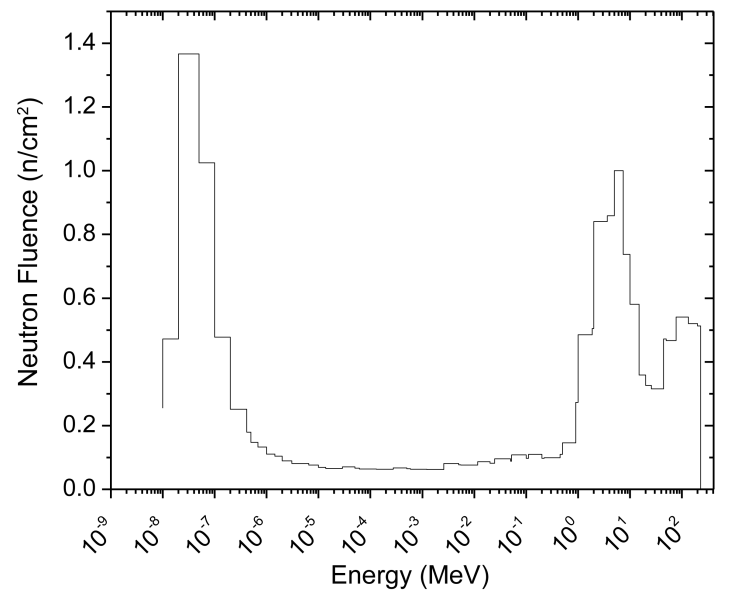

Figure 2: Cosmic-ray induced neutron spectrum measured at Itatiaia Mountain in Brazil.

The ENDF/VI nuclear data library was used for almost all materials. Other libraries were used for materials that do not exist in ENDF. Table 1 describes the 2 other nuclear data libraries that were employed in the simulations.

Nuclear data libraries were used for energies at which they are available for each nuclide and parameterizations corresponding to the MCNPX options given below were used at higher energies:

- Neutron and proton elastic scattering.

- Preequilibrium model after intranuclear cascade.

- Bertini [9] for nucleons and pions and ISABEL [9] for other particles. 
Table 1: Nuclear data libraries for several materials.

\begin{tabular}{ccc} 
& Cadmium & Brass \\
\hline ZAID & $48000.51 \mathrm{c}$ & $30000.42 \mathrm{c}$ \\
Library Name & RMCCS & ENDL92 \\
Date & 1974 & $<1992$ \\
Temp (K) & 293.6 & 300.0 \\
$E_{\max }(\mathrm{MeV})$ & 20 & 30 \\
\hline
\end{tabular}

- Coulomb barrier for incidence charged particles.

- The subtract nuclear recoil energy was not used to get excitation energy.

- Fission was allowed.

- Experimental branching ratios were used.

\section{Results}

Figure 3 shows the behaviour of the angular detector response to CRINS. It is import to note that the angular response was normalized to analyse the behaviour of the LC as function of angle $(\theta)$ between the point source and zenith (figure 1).

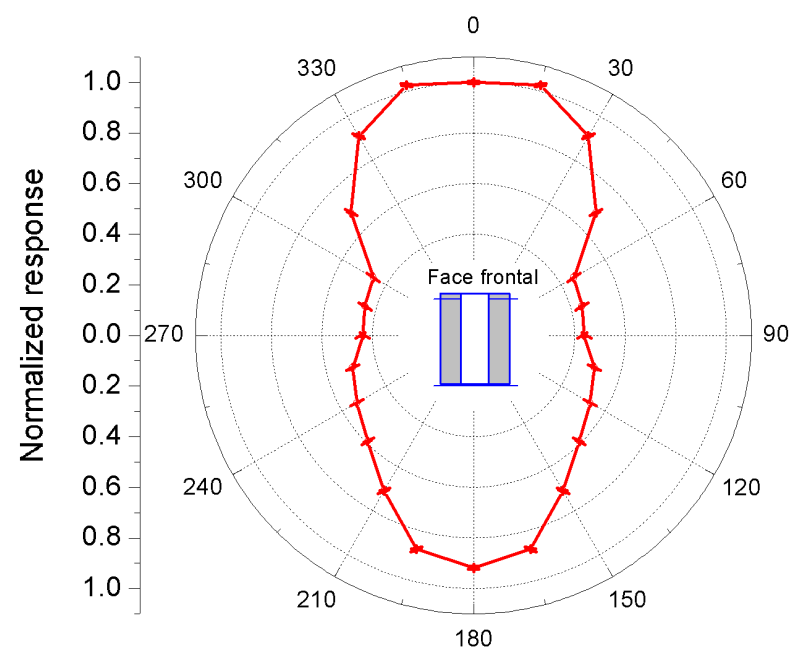

Figure 3: Long Counter response for a point source of a cosmic-ray induced neutron spectrum as a function of the azimuthal angle, obtained using the MCNPX code.

For ground monitoring of the neutron flux resulting from the atmospheric cosmic radiation, the LC must be located with its frontal face directed toward the sky and with its central axis pointed to the zenith. The incident flow is isotropic within a solid angle of $2 \pi$ steradians. In this case, the efficiency of the LC ( $\varepsilon_{\text {isotropic }}$ ) can be related to the efficiency for frontal incidence $\left(\varepsilon_{\text {frontal }}\right)$ by using the angular distribution $f(\theta)$ as: 


$$
\varepsilon_{\text {isotropic }}=\varepsilon_{\text {frontal }} \cdot \int_{0}^{\pi / 2} f(\theta) \cdot \sin (\theta) \cdot d \theta
$$

Table 2 presents the LC efficiency at intervals of $15^{0}$ degrees ranging from 0 to 90 degrees, as well as the response function normalized at $\theta=0^{0}$.

Table 2: LC efficiency for a point source located at various angles relative to the zenith, corresponding to the angular response function normalized at $\theta=0^{0}$

\begin{tabular}{ccc}
\hline Angle & LC efficiency $\left(\right.$ count $\left./ n \cdot \mathrm{cm}^{2}\right)$ & Angular response $(f(\theta))$ \\
\hline 0 & 7.990 & 1.00 \\
15 & 7.995 & 1.00 \\
30 & 6.370 & 0.797 \\
45 & 3.739 & 0.467 \\
60 & 1.896 & 0.237 \\
75 & 1.753 & 0.219 \\
90 & 1.751 & 0.219 \\
\hline
\end{tabular}

By numerically integrating, using the simulated values for $f(\theta)$, we found that:

$$
\varepsilon_{\text {isotropic }}=0.41 \cdot \varepsilon_{\text {frontal }}
$$

In Table 3, a summary of the simulations results and the LC efficiency calculated from experimental measurements [8] is presented.

Table 3: Comparison of LC efficiency by Monte Carlo simulation using point source and analytical integration of a homogeneous source distributed on $2 \pi$ steradians solid angle and experimental results [?]

\begin{tabular}{ccc}
\hline Neutrons from RC & Efficiency $\left(\right.$ count $\left./ \mathrm{n} \cdot \mathrm{cm}^{2}\right)$ & Error $\left(\right.$ count $\left./ \mathrm{n} \cdot \mathrm{cm}^{2}\right)$ \\
\hline Point sources & 3.25 & 0.01 \\
Simulated $(2 \pi$ isotropic source) & 3.22 & 0.03 \\
Measured & 4.95 & 0.70 \\
\hline
\end{tabular}

From Table 3 we see that the agreement between the simulation results is exellent, indicating that both sources of neutrons are consistent with each other. However, these results show a difference with the experimental value of the order of two standard deviations of the measure. This difference can not be immediately assigned to any deviation between the simulation and experimental results, but should be investigated through more precise measurements.

\section{Conclusion}

The results presented here are partial and represent an intermediate stage of the effort for the simulation of a larger virtual environment simulation of cosmic radiation in the atmosphere. At this stage we simulated neutron sources close to the detector to minimize the computation time. However, in this case, the presence of a large volume detector can significantly interfere with the flow of neutrons in their vicinity. 
Another important factor to be considered is the the distribution of fast neutrons in the atmosphere that may not be exactly isotropic [10].

To establish a radiation field, which reproduces the real situation, we are developing a simulation environment of greater extension that considers the propagation of the primary cosmic radiation in the atmosphere, to obtain the thermalized neutrons produced at any altitude. Through this study, we expect to solve the problem of the angular distribution as a function of energy of neutrons that reach the detector, as well as minimize the influence of the detector in regions close to the primary sources of neutrons.

\section{References}

[1] Hanson, A. O. and McKibben, J. L., A Neutron Detector Having Uniform Sensitivity from $10 \mathrm{KeV}$ to 3 MeV, Phys. Rev., vol 72, 673-677, 1947.

[2] Landim,E., Hamburger, E. W., Dietzsch, O., Efficiency of a Long Counter as a Function of the direction and locus of incidence of the teutron, Nuclear Instruments \& Methods, 15, 300-305, 1962.

[3] Tagziria, H., Thomas, D. J., Calibration and Monte Carlo Modeling of neutron Long Counters, Nuclear Instruments and Methods in Physics Research, 452, 470-483, 2000.

[4] Federico, C.A, Oliveira, W.A, Pereira, M.A, Gonçalez, O.L., Avaliação da resposta de um contador do tipo "Long-Counter" para nêutrons de ${ }^{241} \mathrm{Am} / \mathrm{Be}$, Journal of Aer Technol and Management, vol 1, 243-249, 2009.

[5] Pazianotto, M. T., Federico, C. A., Gonçalez, O. L., Calson, B. V.. Evaluation of the Neutron Detector Response for Cosmic Ray Energy Spectrum by Monte Carlo Transport Simulation. In: XIII Workshop on Nuclear Physics, 2011, Havana. WONP-NURT'2011. Havana : InSTEC-CEADEN, 2011.

[6] Pazianotto, M. T., Gonçaliez, O. L., Federico, C. A., Carlson, B. V.. Evaluation of the Response of a Neutron Detector of the Long-Counter Type Using Monte Carlo Transport Simulation. AIP Conference Proceedings, v. 1351, p. 197-201, 2011.

[7] Pelowitz D. MCNPX User's manual version 2.5.0. Los Alamos: National Laboratory; 2005. Report LA-CP-05-0369.

[8] Federico, C.A., Gonçalez, O.L., Fonseca, E.S., et all, Neutron spectra measurements in south Atlantic anomaly region, Rad. Measurements, Vol. 45, 2010, p. 1526-1528.

[9] Baylac-Domengetroy, V., Forschungszentren, H.H.G.D, Investigations related to the generation of reaction products in the target of accelerator driven systems for nuclear waste incineration, Forschungszentrum Karlsrube, 2003.

[10] Frank, M.I., Prussin, S.G., Peterson, P.F., Tobin, M.T., A Monte Carlo model of sea-level neutron background: Directionality, spectra, and intensity, Journal of Radioanalytical and Nuclear Chemistry, Vol. 29, 145-151, 2001. 